\title{
THE SMALL SPECIES OF BELOSTOMA(HETEROPTERA, BELOSTOMATIDAE). I. KEY TO SPECIES GROUPS AND A REVISION OF THE DENTICOLLE GROUP
}

\author{
Ana Lía Estévez ${ }^{1}$ \\ John T. Polhemus ${ }^{2}$
}

\begin{abstract}
A key to species groups of the genus Belostoma Latreille, 1807, using new taxonomic characters are presented as well as the revision of the four species included in the denticolle group: $B$. denticolle Montandon, 1903, and three new species: B. orbiculatum from eastern Argentina and southern Brazil, B. retusum from eastern Argentina and B. amazonum from northern Brazil which are described and illustrated.
\end{abstract}

KEYWORDS. Belostoma, small species, revision, denticolle group.

\section{INTRODUCTION}

The giant water bug genus Belostoma Latreille, 1807, which is Panamerican in distribution, is presently known by about 65 species. Although numerous contributions on taxonomy of the genus are available, those with a monographic criterion are scarse in the last 40 years (De Carlo, 1938; LaucK, 1962, 1963, 1964; SchNaCK, 1976). LaucK (1962) proposed 16 species groups on the basis of the male genitalia and recognized three size categories: large or moderately large species (26.0 to $50.0 \mathrm{~mm}$ in lenght), medium size species (15.5 to $26.0 \mathrm{~mm}$ in lenght) and small species (9.5 to 22.0 in lenght). Lauck's monograph was published in three papers (LAUCK , 1962, 1963, 1964) where he treated large and medium sized species; and named pygmeum, denticolle, oxyurum and plebejum as small species groups, but he did not include any species within them.

The purpose is to offer a new vision of the generic species group composition, based on the treatment of new characters (measure of suture anteclypeus-maxilary plate, measure of suture anteclypeus-loral plate and abdominal pilosity) in combination with the ones anteriorly used. As a result five small species groups are here recognized: triangulum, minor, oxyurum, plebejum and denticolle. The revision of the last mentioned one is also treated here, recognizing three new species.

\section{MATERIAL AND METHODS}

The examined material was borrowed by the following institutions: Francis Huntington Snow Entomological collections, Kansas University, Lawrence (KU); Los Angeles County Museum, Los Angeles (LACM); National Museum of Natural History, Washington D.C. (USNM); The Natural History Museum, London (BMNH); John T. Polhemus collection, Denver (JTPC). The specimens were softened in hot water during 15 minutes in order to remove the genital capsule helped with a pin.

Taxonomic characters: length and width are here presented in $\mathrm{mm}$. Suture anteclypeus-maxillary plate (s') and suture anteclypeus-lorum (s"), both measured on dorsal view (fig.1). Anteoculus (ao), measured from anterior margin of the eyes to anterior tip of the head (fig. 1). Interoculus (io), portion between anterior and

1. Departamento Científico de Entomología, Museo de La Plata, Paseo del Bosque s/n, 1900 La Plata, Argentina.

2. University of Colorado Museum, 3115 S. York St. Englewood, Colorado 80110, USA. 


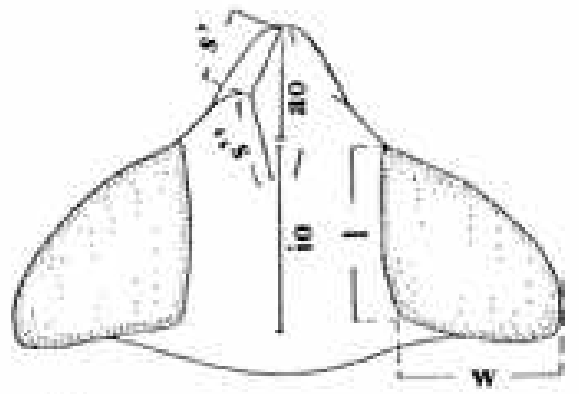

1
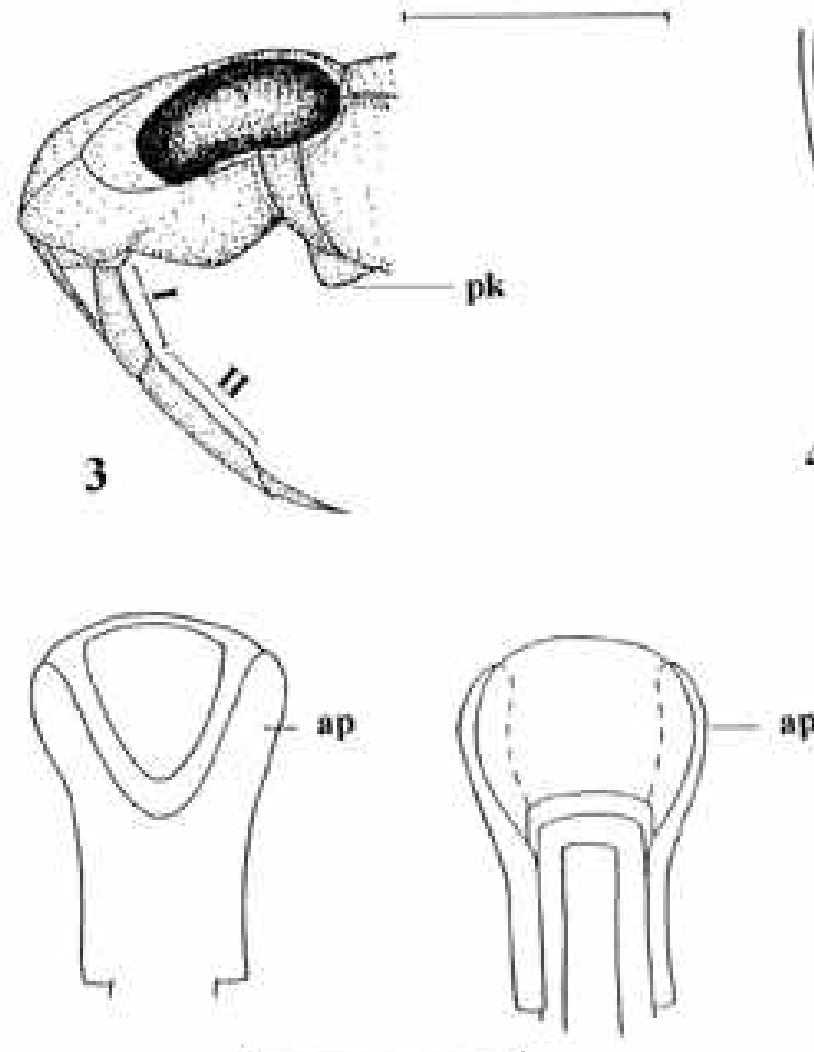

6
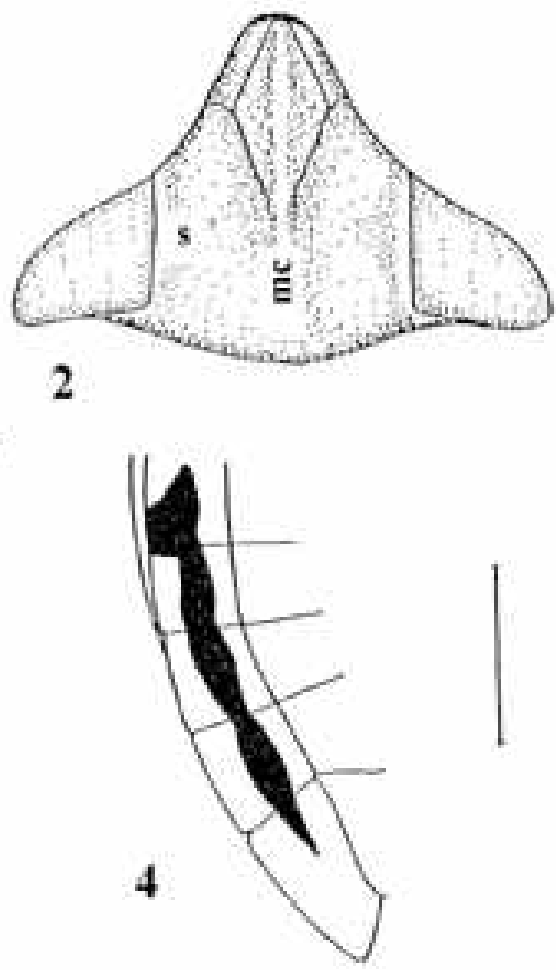

ap

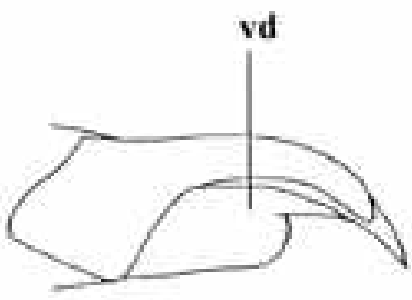

7

Figs. 1-7. Belostoma sp.: 1, 2, head, dorsal and ventral view (ao, anteoculus; io, interoculus; 1, length of eye; mc, median carina; s, sulcus; s', suturae anteclipeus-maxillary plate; s", suturae anteclipeus-loral plate; $w$, width of eye; 3, head, lateral view (I, first segment of the beak; II, second segment of the beak; pk, prosternal keel); 4, abdominal pilosity; 5, male genitalia, dorsal view; 6, ventral; 7, lateral (ap, arms of phallobase; vd, ventral diverticulum). Scale lines: $2 \mathrm{~mm}$, figs. $1-3 ; 3 \mathrm{~mm}$, fig. $4 ; 1 \mathrm{~mm}$, figs. 5,6 . 
posterior margins of eyes (fig.1). Width and length of the eyes were measured (fig. 1). Sulcus (s), a depression along internal margin of the eye (fig. 2). Median carina (mc), longitudinal keel along midline of the head (fig. 2). Beak, lengths of first (I) and second (II) visible segments were measured (fig.3). Prosternal keel (pk), shows three different forms: rounded (prominent or not prominent), acutely pointed (with the anterior margin straight) or triangular shaped (fig. 3). Pilosity, band of hairs that covers part or the entire surface of ventral laterotergites, never covering their outer margin (fig. 4). This stripe was briefly mentioned by De CARLo (1930) and LaUCK (1962). Nevertheless, they used it as diagnostic in specific recognition only in a few species. Pubescence, short hairs covering part or the entire surface of abdominal esternites. Male genitalia, phallobase (pb) and ventral diverticulum (vd) (figs. 5-7).

Key to the groups of the species of Belostoma

1. Pilosity covering entirely ventral laterotergites ................................................... 2

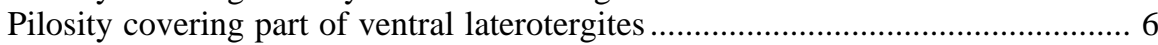

2. North american species .......................................................... B. flumineum group

South american species .................................................................................... 3

3. Pubescence covering part or all of abdominal sternites ........................................ 4

Pubescence not on abdominal sternites ........................................................... 5

4. Eyes triangular ............................................................................ B. bergi group

Eyes globular ............................................................... B. testaceopallidum group

5. Large, dilated species, more than $30.0 \mathrm{~mm}$ long ...................... B. dilatatum group Less than 30.0 long ........................................................ B. bifoveolatum group

6. Pronotum and scutellum with a distinct median carina ........ B. aurivilianum group

Pronotum and scutellum without median carina ................................................. 7

7. Outer margin of eye straight, eye triangular ........................................................ 8

Outer margin of eye rounded, eye globose …................................................. 9

8. Segment I of the beak longer than II ............................................. B. bergi group

Segment I of the beak shorter than II ....................................... B. discretum group

9. Small species, less than $24.0 \mathrm{~mm}$ long; segment I of the beak shorter than II ... 10 Larger species, more than $28.0 \mathrm{~mm}$ long; segment I of the beak longer or equal

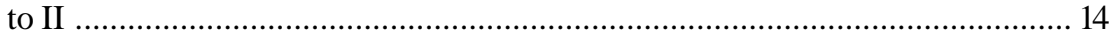

10. Prosternal keel rounded or acutely pointed, projecting anterad ............................. 11 Prosternal keel triangular shaped, not projected anterad .......... B. triangulum group

11. Pilosity covering more than half of ventral laterotergites ...................................... 12

Pilosity covering half or less of ventral laterotergites ......................................... 13

12. Sulcus covered with short and dense pilosity that extends from behind eyes to lorum B. minor group Sulcus covered with short and scattered pilosity that extends along eyes

B. oxyurum group

13. Prosternal keel rounded or with the anterior margin straigth, not projeted anterad; pilosity slightly developed

B. plebejum group Prosternal keel acutely pointed or rounded projected anterad; pilosity well developed B. denticolle group

14. Vertex with prominent carina; scutellum not reaching nodal line ..................... 15

Vertex without a median carina; scutellum reaching nodal line ...................... 16

15. Clypeus reaching ocular line .......................................................... B. stolli group Clypeus not reaching ocular line ........................................... B. dilatatum group 
16. Ventral diverticulum of male genitalia with a very large distinct apicoventral protuberance ..................................................................... B. dentatum group

Ventral diverticulum of male genitalia with moderately apicoventral protuberance B. subspinosum group

\section{Belostoma denticolle group}

Size: length, 11.0-16.0; width, 5.0-7.0. Shape: ovate. Color: light to dark brown. Head: suture anteclypeu-maxilary plate as long as suture anteclypeus lorum; maxillary plate compressed or not compressed; anteoculus shorter than interoculus; eyes globose, as wide as long or wider than long; segment I of the beak as long or shorter than II. Thorax: prosternal keel prominent, acutely pointed or rounded and projected anterad. Abdomen: pilosity well developed, covering a moderately wide band on ventral laterotergites, slightly constricted between spiracles.

Male genitalia: ventral diverticulum flat, arms of phallobase longer than posterior portion of phallobase.

Observation: this group resembles oxyurum group by the flat shape of the male genitalia, but differs in that pilosity is narrower than in oxyurum group.

Key to the species of denticolle group

1. Prosternal keel acutely pointed, prominent, projecting anterad ............................. 2 Prosternal keel rounded, prominent .................................. B. orbiculatum sp. nov.

2. Maxillary plate not compressed; prosternal keelstrongly developed ....................... 3 Maxillary plate compressed; prosternal keel not strongly developed

B. retusum sp. nov.

3. Suture anteclypeus-maxillary plate slightly longer than suture anteclypeus-lorum B. denticolle Montandon

Suture anteclypeus-maxillary plate as long as suture anteclypeus-lorum B. amazonum sp. nov.

\section{Belostoma orbiculatum sp. nov.}

(Figs. 8-10, 20-23)

Size: length, $0^{\top 13.4-14.6, ~} 13.55-14.7$; width, $o^{\top} 6.7-7.5$, , 6.7-7.6. Color: almost uniformly brown. Shape: elliptical (fig. 8).

Head: suture anteclypeus-maxilary plate as long as suture anteclypeus-lorum; maxillary plate not compressed; anteoculus shorter than interoculus; eyes globose, as wide as long (fig. 9); segment I of the beak slightly shorter than II (fig. 10). Thorax: prosternal keel prominent, rounded (fig. 10). Abdomen: pilosity slightly developed on penultimate segment (fig. 20).

Male genitalia: arms of phallobase covering lateral margins of ventral diverticulum (figs. 21-23).

Diagnosis. Belostoma orbiculatum is easily distinguished from the other species of the group in having the prosternal keel rounded.

Distribution. Southeastern and South Brazil, Argentina and Uruguay. 
Types. Holotype $\sigma^{7}$, allotype $q(\mathrm{KU}) ; 7$ paratypes $\left(4 \sigma^{\prime}, 3\right.$ 9) labelled "Argentina, Bs. As., Tigre, VI-14-'69, P. \& P. Spangler" (USNM); paratype ơ from Buenos Aires, Argentina (USNM).

Other specimens examined. BRAZIL, Rio de Janeiro: Rio de Janeiro, q, Wygodzinsky coll. (JTPC); Santa Catarina: Nova Teutonia, + , VIII.1932/I.1933, Johnk \& Holst coll. (JTPC); O’, XI.1966, Plaumann coll. (LACM). ARGENTINA, Buenos Aires: Tigre, 6 ơ, 4 9, 14.VI.1969, P. \& P. Spangler coll. (USNM); Buenos Aires, $O^{\top}$ (USNM). URUGUAY, Cerro Largo: Sarandí del Quebracho, ơ, XI.1959, Carbonell et al. coll. (LACM).

\section{Belostoma retusum sp. nov.}

(Figs. 11-13, 24-27)

Size: length: $o^{7}$ 11.0-12.0, o 11.5-12.0; width: $\sigma^{\top}$ 5.0-5.5, q 5.0-6.0 mm. Color: brown. Shape: elliptical, elongate (fig. 11).

Head: suture anteclypeus-maxilary plate as long as suture anteclypeus-lorum; maxillary plate strongly compressed; anteoculus as long as interoculus; eyes globose, wider than long (fig. 12); segment I of the beak as long as II (fig. 13). Thorax: prosternal keel acutely pointed, slightly projected anterad (fig. 13). Abdomen: pilosity well developed on penultimate segment (fig. 24).

Male genitalia: arms of phallobase straight not covering lateral margins of ventral diverticulum (figs. 25-27).

Diagnosis. Belostoma retusum is easily distinguished from the other species of the group in having the maxillary plate strongly compressed.

Distribution. Villarica (Paraguay) and Manantiales, Corrientes (Argentina).

Types. Holotype $\sigma^{\top}$, alotype,+ 22 paratypes $\left(3 \sigma^{\top}, 19 \%\right)$ are labeled: "Paraguay S.A., Villarica, 12-29 1929, F. Schade" (KU); $20^{\prime}$, paratypes, idem (JTPC).

Other specimens examined. PARAGUAY, Villarica, $20^{\prime}$, ,, XII.1929, Schade coll. (JTPC). ARGENTINA, Corrientes: Manantiales,,+ , 15-30.I.1966 (JTPC).

\section{Belostoma denticolle Montandon}

(Figs. 14-16, 28-31)

Belostoma denticolle Montandon, 1903:362; NIESER, 1975:113; LANZER- DE- SouZA, 1980:52, 53.

?Belostoma denticolle; DE CARLO, 1938:226 (record from Amazonas, questionable).

Belostoma candidulum; DE CARLO, 1957:55 (in part).

Size: length: o 12.0 - 14.0, $\$ 12.0$ - 14.0; width: o $^{7} 5.5$ - 6.0, $q 5.5$ - 6.0. Color: light brown to dark brown. Shape: ovoid, elongate (fig. 14).

Head: suture anteclypeus-maxilary plate longer than suture anteclypeus-lorum; maxillary plate slightly compressed; anteoculus distinctly shorter than interoculus; eyes globose, as wide as long (fig. 15); segment I of the beak shorter than II (fig. 16). Thorax: prosternal keel acutely pointed, prominent (fig. 16). Abdomen: pilosity slighty developed on penultimate segment (fig. 28).

Male genitalia: arms of phallobase divergent, covering lateral margins of ventral diverticulum, one and one half times longer than posterior part of phallobase (figs. 29-31).

Diagnosis. Belostoma denticolle differs from others of the group by the suture anteclypeus-maxillary plate longer than suture anteclypeus-lorum.

Distribution. Northern of South America (Venezuela and Guyana).

Types. We haven't seen the cotype series established by MonTANDOn (1903) from “Amer. mer. Surinam”, deposited in the Hungarian National Museum. 

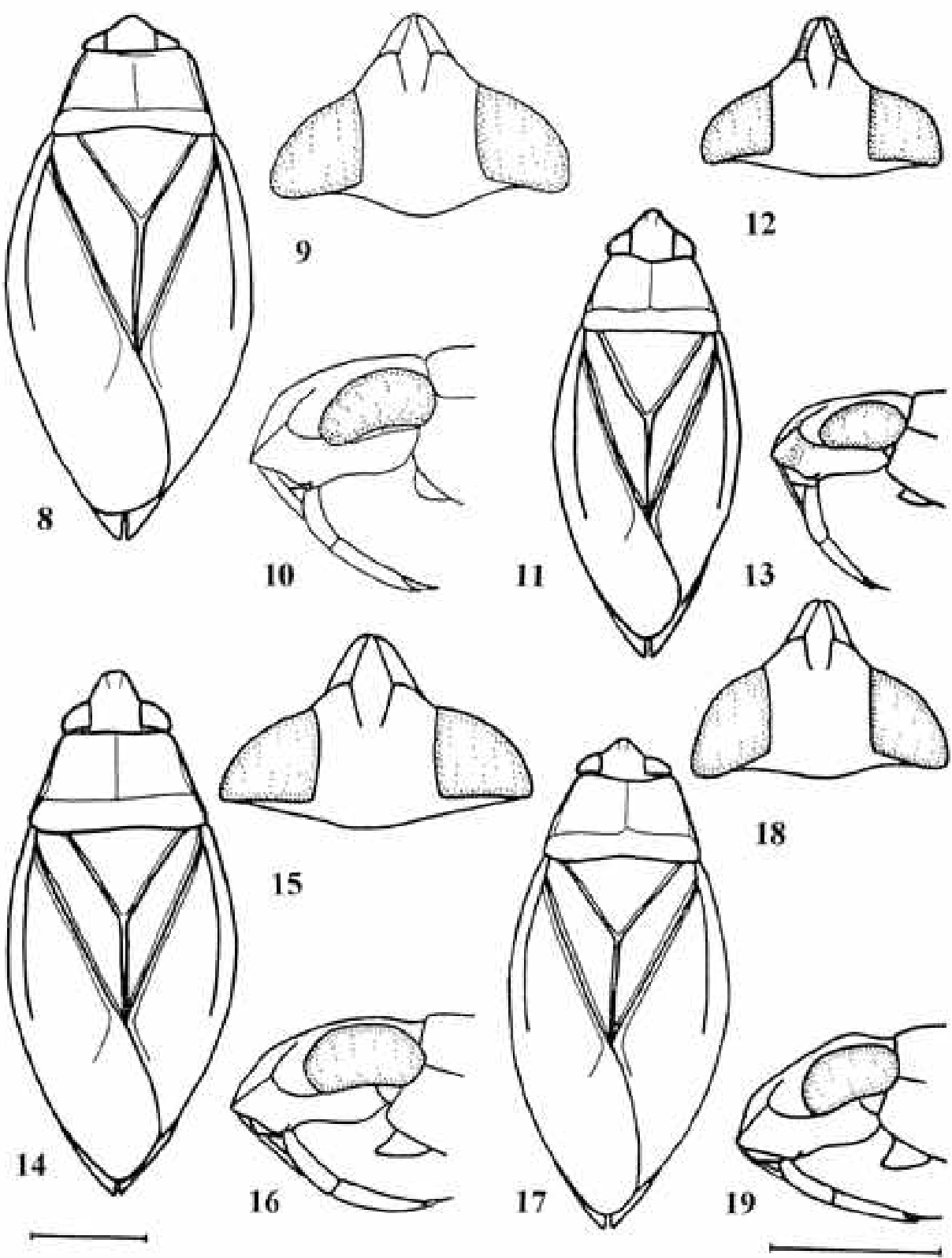

Figs. 8-19. Body form, head dorsal and lateral views. 8-10, Belostoma orbiculatum sp. nov.; 11-13, B. retusum sp. nov.; 14-16, B. denticolle Montandon; 17-19, B. amazonum sp. nov. Scale lines: 3 mm, figs. 8, 11, 14, 17; 2 mm; figs. $9,10,12,13,15,16,18,19$. 

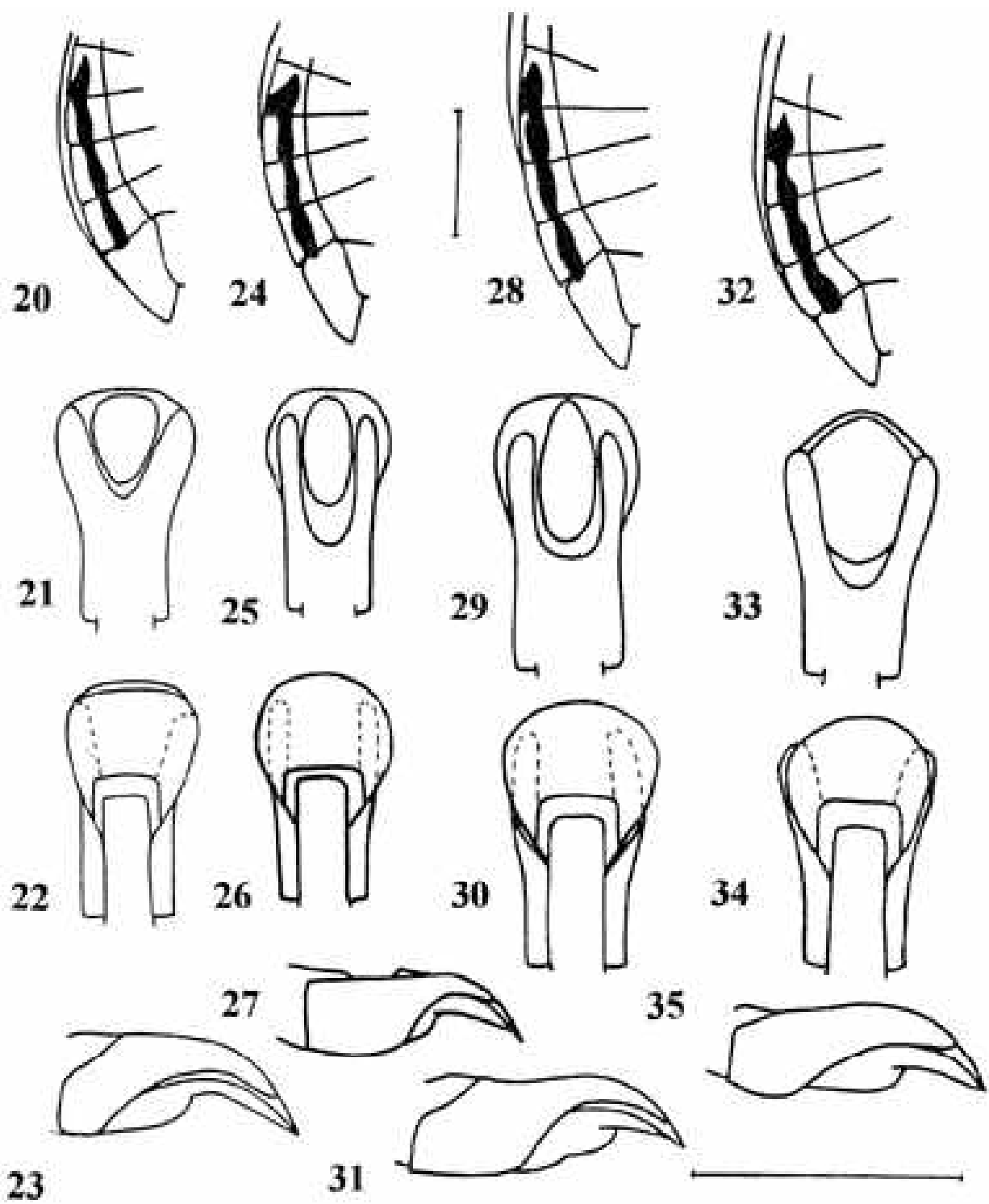

Figs. 20-35. Abdominal pilosity and male genitalia dorsal, ventral and lateral views: 20-23, Belostoma orbiculatum sp. nov.; 24-27, B. retusum sp. nov.; 28-31, B. denticolle Montandon; 32-35, B. amazonum sp. nov. Scale line: 1 mm, figs. 20-35. 
Specimens examined. GUYANA, Supuruni Creek, $2 \sigma^{\top}$,, , Harris coll. (JTPC); Lamaha E coast, Demerara,,, 1932 , Harris coll. (JTPC). VENEZUELA, Monagas: Maturín, 5 O, 5 ९,1958, Menke coll. (JTPC); Caracas, 2 \%, 1950, Roze coll., (JTPC). Without locality references, ơ (BMNH).

\section{Belostoma amazonum sp. nov.}

(Figs. 17-19, 32-35)

Size: length: ${ }^{\top}$ 13.0-15.0,, $14.5-16.0$; width: ${ }^{\top} 6.0-6.5, q 6.0-7.0$. Color: light brown. Shape: ovoid, elongate (fig. 17).

Head: suture anteclypeus-maxilary plate as long as suture anteclypeus lorum; maxillary plate not compressed; anteoculus slightly shorter than interoculus; eyes globose, as wide as long (fig. 18); segment I of the beak shorter than II (fig. 19). Thorax: prosternal keel acutely pointed, prominent (fig. 19). Abdomen: pilosity well developed on penultimate segment (fig. 32).

Male genitalia: arms of phallobase straight, not covering lateral margins of ventral diverticulum (figs. 33-35).

Diagnosis. Belostoma amazonum is similar to $B$. denticolle but differs in head and in the arms of phallobase do not cover lateral margins of ventral diverticulum.

Distribution. Apparently restricted to the drainage basin of Amazonas River.

Types. Holotype $\sigma^{\alpha}$, allotype,+ 45 paratypes $\left(200^{\alpha}, 25\right.$ ? $)$ bearing the label: "Manacapuru S.A., Amazonas, Brasil, Solimões River, 6-26, S.M. Kinges” (KU); 3 paratypes (20', ㅇ), same locality (JTPC).

Other specimens examined. VENEZUELA, Guar. Calabozo, $30^{\circ}, 3 \%$, 1969, Spangler coll. (JTPC). BRAZIL, Amazonas: ( (BMNH); Bahia: Santa Rita de Cassia, Rio Preto, $50^{\prime}, 4$, IV.1958, Dente coll. (LACM); Santa Catarina: Corupa, $20^{\top}$, IX.1948, Maller coll. (BMNH). Without locality references, $0^{\top}(\mathrm{BMNH})$.

Acknowledgments. To Dr. David Lauck for encoraging us to continue with his work.

\section{REFERENCES}

De CARlo, J. A. 1930. Familia Belostomatidae, géneros y especies para la Argentina. Revta Soc. ent. argent., Buenos Aires, 13:101-124.

1938. Los Belostómidos americanos (Hemiptera). An. Mus. argent. Cienc. nat., Buenos Aires, 39:189-252.

-. 1957. Especies nuevas de la familia Belostomatidae (Hemiptera) y algunas consideraciones sobre otras poco conocidas. Revta Soc. ent. argent., Buenos Aires, 19: 47-59.

LANZER-DE-SouZA, M. E. 1980. Inventário da distribuição geográfica da família Belostomatidae Leach, 1815 (Hemiptera-Heteroptera) na Região Neotropical. Iheringia, Sér. Zool., Porto Alegre, (55):43-86.

LaUCK, D. R. 1962. A monograph of the genus Belostoma (Hemiptera). Part I. Introduction and B. dentatum and subspinosum groups. Bull. Chicago Acad. Sci., Chicago, 11(3):34-81.

_. 1963. A monograph of the genus Belostoma. Part II. B. aurivilianum, stolli, testaceopallidum, dilatatum and discretum groups. Bull. Chicago Acad. Sci., Chicago, 11 (4):82-101.

- 1964. A monograph of the genus Belostoma. Part III. B. triangulum, bergi, minor, bifoveolatum and flumineum groups. Bull. Chicago Acad. Sci., Chicago, 11 (5):102-154.

Montandon, A. L. 1903. Especes nouvelles ou peu connues du genre Belostoma. Appartennant aux collections du Musée National Hongrois. Ann. Mus. Nat. Hungr., 1:359-363.

Nieser, N. 1975. The water bugs (Heteroptera-Nepomorpha) of the Guyana Region. Stud. Fauna Suriname, Utrecht, 16(59):1-310.

Schnack, J. A. 1976. Insecta-Hemiptera-Belostomatidae. Fauna Agua Dulce Rep. Arg., Buenos Aires, 35(1):1-66.

Recebido em 28.01.2000; aceito em 13.07.2001 